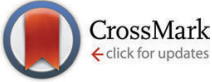

Cite this: Phys. Chem. Chem. Phys., 2017, 19, 14376

Received 13th January 2017 Accepted 28th February 2017 DOI: $10.1039 / c 7 c p 00265 c$ rsc.li/pccp

\section{Local $\mathrm{pH}$ and effective $\mathrm{p} K_{\mathrm{A}}$ of weak polyelectrolytes - insights from computer simulations $\dagger$}

\begin{abstract}
Lucie Nová, Filip Uhlík and Peter Košovan*
In this work we study the titration behavior of weak polyelectrolytes by computer simulations. We analyze the local pH near the chains at various conditions and provide molecular-level insight which complements the recent experimental determination of this quantity. Next, we analyze the non-ideal titration behaviour of weak polyelectrolytes in solution, calculate the effective ionization constant and compare the simulation results with theoretical predictions. In contrast with the universal behaviour with respect to chain length, we find non-universality and deviations from theory with respect to polymer concentration and permittivity of the solvent. The latter we explain in terms of counterion condensation and ion correlation effects, which lead to reversal of the non-ideal titration behaviour at very low permittivities. We discuss the impact of these findings on the interpretation of experimental results.
\end{abstract}

\section{Introduction}

Ionizable groups in weak polyelectrolytes are weak acids or bases, and their ionization is variable in response to changes in the environment. This is in contrast with strong polyelectrolytes, ionization of which is fixed at the time of synthesis. Compared to low molar mass weak acids and bases, dissociation of weak polyelectrolytes is much more complex. The electrostatic interaction between the nearby ionized groups on the chain provides an additional conformation-dependent free energy cost of ionization. Therefore ionization of weak polyelectrolytes is suppressed as compared to the corresponding monomer. In addition, the increased ionization of the macromolecule brings about expansion of its conformation, and changes in the distribution of counter-ions and of other small ions around the chain.

The variable ionization of weak polyelectrolytes is being exploited in various $\mathrm{pH}$-responsive polymer systems. For example, the pH-dependent self-assembly and gelation of diblock copolymers ${ }^{1-3}$ and of gradient copolymers ${ }^{4,5}$ containing poly(acrylic acid), pH-responsive swelling and collapse of polymer-coated nanoparticles, ${ }^{6}$ polyelectrolyte microgels ${ }^{7,8}$ and co-networks. ${ }^{9,10}$ However, in most experimental works the interpretation of $\mathrm{pH}$-responsive behaviour of weak polyelectrolytes is based on intuitive extrapolation of the textbook knowledge about ionization of simple weak acids. For example, it is commonly

Department of Physical and Macromolecular Chemistry, Faculty of Science,

Charles University, Hlavova 8, 128 oo Praha 2, Czech Republic.

E-mail:peter.kosovan@natur.cuni.cz

$\dagger$ Electronic supplementary information (ESI) available. See DOI: 10.1039/c7cp00265c assumed that at $\mathrm{pH}>\mathrm{p} K_{\mathrm{A}}+1$ the acid is almost fully ionized. This is true for low-molecular acids while polyelectrolytes hardly reach $50 \%$ ionization under such conditions.

In this work we use computer simulations to unravel some of the non-trivial features of the non-ideal ionization of weak polyelectrolytes in solution. We address the local effects that arise as a consequence of coupling between ionization and conformation of the polyelectrolyte. We also address the changes in titration curves of the polyelectrolyte with varying chain length, polymer concentration, and solvent permittivity.

\section{Theoretical background}

\subsection{Thermodynamics of acid-base equilibria}

Some well-known terms from theory of acid-base equilibria attain non-trivial meanings in nano-heterogeneous systems, such as polyelectrolyte solutions. Therefore we briefly review here the key points of the theory, emphasizing the connection to polyelectrolytes. For simplicity, we restrict further discussion to weak polyacids, keeping in mind that analogous arguments apply to polybases. Consider the schematic ionization reaction of a weak acidic group A

$$
\mathrm{HA} \leftrightharpoons \mathrm{A}^{-}+\mathrm{H}^{+}
$$

The reaction equilibrium in eqn (1) is characterized by the acidity constant $K_{\mathrm{A}}$, or its negative $\operatorname{logarithm~} \mathrm{p} K_{\mathrm{A}}=-\log _{10} K_{\mathrm{A}}$, which is related to the chemical potentials as

$$
k_{\mathrm{B}} T \ln K_{\mathrm{A}}=\sum_{i} \nu_{i}\left(\mu_{i}-\mu_{i}^{\ominus}\right),
$$


where $\Delta_{\mathrm{r}} G^{\ominus}=\sum_{i} \nu_{i} \mu_{i}^{\ominus}$ is the standard Gibbs free energy change of the reaction, $\nu_{i}$ is the stoichiometric coefficient and $\mu_{i}$ the chemical potential of species $i$ participating in the reaction. The chemical potential can be further written in terms of activity

$$
\mu_{i}-\mu_{i}^{\ominus}=k_{\mathrm{B}} T \ln a_{i}=k_{\mathrm{B}} T \ln \left(\frac{c_{i}}{c^{\ominus}}\right)+k_{\mathrm{B}} T \ln \left(\gamma_{i}\right),
$$

where $\mu_{i}^{\ominus}$ is the reference (standard) chemical potential, $a_{i}$ is the activity, $c_{i}$ is the concentration, $\gamma_{i}$ is the activity coefficient of species $i$. The reference concentration $c^{\ominus}$ is given by the choice of the reference state. We can further split the chemical potential to the ideal gas term, $\mu_{i}^{\text {id }}$, and the excess term, $\mu_{i}^{\text {ex }}$, which accounts for the influence of interactions:

$$
\mu_{i}^{\mathrm{id}}=k_{\mathrm{B}} T \ln \left(c_{i} / c^{\ominus}\right), \mu_{i}^{\mathrm{ex}}=k_{\mathrm{B}} T \ln \left(\gamma_{i}\right)
$$

It is convenient to characterize ionization in terms of the degree of ionization - the ratio of the amount of the ionized form and the total amount of the given species,

$$
\alpha=c_{\mathrm{A}^{-}} /\left(c_{\mathrm{A}^{-}}+c_{\mathrm{HA}}\right) \text {. }
$$

Substituting from eqn (5) and (3) to eqn (2) and assuming ideal behaviour $(\gamma=1)$, we obtain the ideal titration curve

$$
1 / \alpha=1+10^{\mathrm{p} K_{\mathrm{A}}-\mathrm{pH}},
$$

which well describes ionization of simple low-molar-mass acids up to $c \approx 0.1 \mathrm{M}$. Eqn (6) is often used as the defining relation for the effective acidity constant, $\mathrm{p} K_{\text {eff }}^{11-13}$

$$
\mathrm{p} K_{\mathrm{eff}} \equiv \mathrm{p} K_{\mathrm{A}}+\log _{10}\left(\frac{\gamma_{\mathrm{H}^{+}}}{\gamma_{\mathrm{A}^{-}}}\right)=\mathrm{pH}+\log _{10}\left(\frac{1-\alpha}{\alpha}\right),
$$

which can be calculated from the experimentally measured $\alpha$. Because $\mathrm{p} K_{\text {eff }}$ implicitly includes all the non-idealities, it is not a true constant but exhibits a non-trivial dependence on all relevant parameters, such as the degree of ionization, polymer chain length, polymer concentration and salt concentration. ${ }^{12,14,15}$ Consequently, it characterizes one particular state of the system under one specific set of conditions. Although the use of $K_{\text {eff }}$ is convenient from practical view of analyzing experimental data, it only provides limited information for understanding of the underlying phenomena and for predictions of behaviour under different conditions.

\subsection{The definition of $\mathrm{pH}$}

In eqn (7) we have assumed the following definition of $\mathrm{pH}$

$$
\mathrm{pH}(\mathbf{r}) \equiv-\log _{10} \frac{c_{\mathrm{H}^{+}}(\mathbf{r})}{c^{\ominus}}=-\log _{10} \frac{a_{\mathrm{H}^{+}}}{\gamma_{\mathrm{H}^{+}}(\mathbf{r})},
$$

which allows us to use the term "local $\mathrm{pH}^{\mathrm{H}}$ " as it is commonly used in literature to describe local variations in $c_{\mathrm{H}^{+}}(\mathbf{r})$ in nanoheterogeneous systems. To emphasize this, we have explicitly written the relevant quantities in eqn (8) as a function of the position vector r. Local $\mathrm{pH}$ is often invoked to explain deviations from the ideal behaviour but it is very difficult to measure. Nevertheless, it has been recently measured for a single polyelectrolyte chain and for a star-like polyelectrolyte in solution by means of single-molecule fluorescence techniques. ${ }^{16-18}$
In this context it is noteworthy that historically the $\mathrm{pH}$ has been first defined by means of concentration of $\mathrm{H}^{+}$ions, ${ }^{19}$ and only later it has been defined by means of the activity. ${ }^{20,21}$ The present operational definition $^{21}$ relies on a convention based on the electromotive force on the hydrogen (or glass) electrode, such that it can be reproducibly measured and is close to $-\log _{10} a_{\mathrm{H}^{+}}{ }^{20}$ Note that the chemical potential and activity are constants throughout the whole system, and do not vary locally under equilibrium. If there are local variations of concentration, these have to be compensated by local variations of the activity coefficient, as is implied by eqn (3) and (8). Note that if $\mathrm{pH}$ should be defined as the logarithm of activity, rather than concentration, it implies that at equilibrium $\mathrm{pH}$ is constant in the whole system, and the term "local $\mathrm{pH}$ " would become essentially meaningless. In further text, we will use the term local $\mathrm{pH}$ for $\mathrm{pH}$ in the immediate vicinity of the chain (see section Local $\mathrm{pH}$ and bulk $\mathrm{pH}$ for more precise definition). By bulk $p H$ we will refer to $\mathrm{pH}$ far from the chain, where the influence of the polyelectrolyte is minimal.

\subsection{Theory of ionization of weak polyelectrolytes}

Theoretical attempts to predict the ionization degree of a weak polyelectrolyte as a function of its molecular weight, concentration, solution $\mathrm{pH}$, or permittivity can be tracked back to the seminal work of Katchalsky and Gillis. ${ }^{14}$ Within the Debye-Hückel approximation for electrostatic interaction, assuming charges homogeneously distributed along the chain, and neglecting correlations with counterions they derived the following equation:

$$
\mathrm{pH}-\mathrm{p} K_{\mathrm{A}}=-\log _{10} \frac{1-\alpha}{\alpha}+A \lambda\left(\frac{3 \alpha}{\lambda^{\prime} k j}\right)^{\frac{1}{3}}\left(\frac{l_{\mathrm{B}}}{b}\right)^{\frac{2}{3}}
$$

where $l_{\mathrm{B}}$ is the Bjerrum length defined in eqn (13), $b$ is the bond length, $A=2(4 \pi)^{2 / 3} \ln (10)$ is a constant pre-factor, $k$ is the number of monomers per Kuhn segment, and $1 / j$ is the fraction of monomers of the polymer capable of ionization. The parameters $\lambda$ and $\lambda^{\prime}$ are defined as

$$
\lambda=1+\ln \frac{3 R_{\mathrm{e}}^{2}(\alpha)}{2 R_{0}^{2}}, \quad \lambda^{\prime}=\ln \frac{3 R_{\mathrm{e}}^{2}(\alpha)}{2 R_{0}^{2}}-1,
$$

where $R_{\mathrm{e}}$ and $R_{0}$ are the end-to-end distances of the ionized chain, and of the neutral chain, respectively. Katchalsky and Gillis in their work neglected the interaction between the ionized macromolecules and their counterions and also between different chains. Therefore their theory cannot predict the concentration dependence of the ionization and it should fail at high concentrations and above the Manning condensation threshold. However, as follows from the discussion below, it qualitatively well describes the experimentally observed trends in ionization of polyelectrolytes in a rather broad range of parameters.

Eqn (9) can be further simplified, realizing that $\lambda$ and $\lambda^{\prime}$ vary very slowly with $\alpha$ or $N$. If they are assumed constant, we obtain an explicit relation for the dependence of $\mathrm{p} K_{\text {eff }}$ on $\alpha$

$$
\mathrm{p} K_{\mathrm{eff}}(\alpha, N, \ldots)=\mathrm{p} K_{\mathrm{A}}+m(N, \ldots) \alpha^{1 / 3} u^{2 / 3}
$$


where $u=l_{\mathrm{B}} / b$ is the dimensionless electrostatic coupling parameter, and $m$ is a weakly varying function of all other parameters, which can be determined by fitting experimental data. If both $m$ and $\mathrm{p} K_{\mathrm{A}}$ are treated as adjustable parameters, eqn (11) well represents experimental data for poly(acrylic acid) at low concentrations in salt-free solutions, and the obtained values of $m$ and $\mathrm{p} K_{\mathrm{A}}$ slightly vary with polymer concentration. ${ }^{12}$ This variation of $\mathrm{p} K_{\mathrm{A}}$ reflects the fact that the theory behind eqn (9) does not explicitly account for the influence of counterions, or for interactions between individual polymer chains. Interestingly, for the more hydrophobic poly(methacrylic acid), the use of eqn (11) fails even on the qualitative level. ${ }^{12}$ Presumably this is because hydrophobic polyelectrolytes exhibit domain formation $^{22,23}$ which was not considered in the original theory.

Raphael and Joanny ${ }^{24}$ have been able to predict the local variation of the degree of ionization, $\alpha(s)$, with the monomer position in the chain, $s$, as a function of the average degree of ionization, $\alpha$. Their prediction is in excellent agreement with our recent simulation results, ${ }^{25}$ however, it does not predict how $\alpha$ depends on the properties of the polymer, or of the solution.

Over the years many other phenomenological approaches to predictions of the ionization of weak polyelectrolytes have appeared in literature. However, often the physical meaning of their parameters is not straightforward, or they do not provide a simple analytical relation but require numerical solution of the equations, as discussed in ref. 26 for the special case of poly(acrylic acid). A handful of simulation studies dealing with various aspects of weak polyelectrolytes can also be found in literature. A comprehensive overview can be found in reviews. ${ }^{26-28}$ Below we address some selected papers relevant for further discussion. Early simulations of weak polyelectrolytes have focused on the role of chain length in ionization, and comparison with the universal titration curve..$^{29-31}$ About two decades later, Panagiotopoulos investigated in simulations the role of electrostatic coupling. ${ }^{32}$ Uyaver and Seidel have investigated the collapse of weak polyelectrolytes under poor solvent conditions $^{33,34}$ and the nonuniform charge distribution along the chain ${ }^{35}$ within the Debye-Hückel approximation. The same approximation has been used by Ullner and Jönsson, ${ }^{36,37}$ and by Ulrich et al. to study conformation changes with ionization ${ }^{38}$ and by Carnal et al. to study adsorption of weak polyelectrolytes at nanoparticles. ${ }^{39}$ Other groups have investigated the behaviour of weak polyelectrolyte gels under various conditions. ${ }^{40-42}$ Ziebarth and Wang studied the changes of ionization of poly(ethylene imine) interacting with DNA. ${ }^{43,44}$ In all these studies one of the key aspects of the observed behaviour was the coupling between the conformation and ionization of the weak polyelectrolyte. However, investigation of the local $\mathrm{pH}$ near polyelectrolyte chain by means of computer simulations is lacking. Since the local $\mathrm{pH}$ in polyelectrolyte solutions has been measured in recent experiments, ${ }^{17,18,45,46}$ it is timely to investigate it in computer simulations and to contrast the experimental and simulation results. Similarly, the role of polymer concentration has not been investigated in computer simulations, while its influence on the titration behaviour of weak polyelectrolytes is evidenced in experiments. ${ }^{13}$ The above two aspects of weak polyelectrolyte behaviour are the subject of our present investigation.

\section{Polymer model}

The model and methodology employed in this study are identical to what has been described in our previous publications, ${ }^{25,47}$ therefore we restrict ourselves here to a brief description, and refer the reader to the earlier publications for additional details.

We employ a bead-spring model consisting of $N$ monomers, where each monomer is modeled by a soft repulsive potential which corresponds to the athermal (good) solvent conditions:

$$
U(r ; \varepsilon, c)=\varepsilon k_{\mathrm{B}} T \frac{(r-c)^{2}}{r^{2}},
$$

where $c$ and $\varepsilon$ are adjustable parameters which we set to $\varepsilon=1.5 k_{\mathrm{B}} T$, and $c=2 b$, where $b=0.41 \mathrm{~nm}$ is the effective monomer size. Monomers are connected by harmonic springs with the equilibrium bond length $b$, and the stiffness constant $k=24.4 k_{\mathrm{B}} T \mathrm{~nm}^{-1}$. The solvent is treated implicitly as a dielectric continuum with the given relative permittivity, $\varepsilon_{\mathrm{r}}$, and with the corresponding Bjerrum length:

$$
l_{\mathrm{B}}=\frac{e^{2}}{4 \pi \varepsilon_{0} \varepsilon_{\mathrm{r}} k_{\mathrm{B}} T} .
$$

The charged particles interact via Coulomb potentials and the electrostatic interaction energy is evaluated using the Ewald summation.

We define the degree of ionization of the chain, $\alpha$, as the average over individual monomers:

$$
\alpha=\frac{1}{N} \sum_{i=1}^{N}\left|z_{i}\right|,
$$

where $z_{i}$ is the charge number of monomer $i$ in a particular state. The reported values of $\alpha$ (and all other observables) are estimates of ensemble averages calculated over many system configurations. Each monomer is a weak acid, characterized by the ionization constant, $K_{\mathrm{A}}$, which is the input parameter of the simulation. We carry out a series of simulations, systematically varying $\mathrm{p} K_{\mathrm{A}}$, which determines the value of $\alpha$. We would like to emphasize that we vary $\mathrm{pH}$ by means of varying the strength of the acid, the $\mathrm{p} K_{\mathrm{A}}$ (see Section 4 for more details). No salt was added.

\subsection{Parameters of the studied systems}

Unless stated otherwise, we use the following default set of parameters: number of chains $n=1$ of length $N=100$ beads in a cubic simulation box with edge length $L$ such that the monomer density $c_{\text {pol }}=n N / L^{3} \approx 1.4 \times 10^{-2} \mathrm{~mol} \mathrm{l}^{-1}$. We set the default permittivity to that of water at room temperature, $\varepsilon_{\mathrm{r}}=80$, which results in the electrostatic coupling $u=l_{\mathrm{B}} / b \approx 2$. We systematically vary $K_{\mathrm{A}}$ in different systems such that it covers the whole range of ionization degrees $0<\alpha<1$. To study the effect of molecular weight of the polymer, we vary the length between $1<N<200$. 
When studying the concentration effects, we vary the simulation box size such that the concentration of monomer units is in the range $1.4 \times 10^{-2}<c_{\text {pol }}<1.4 \times 10^{-1} \mathrm{~mol} \mathrm{l}^{-1}$. For poly(acrylic acid) this corresponds to the usual concentration range used in experiments, $1.0-10.0 \mathrm{mg} \mathrm{ml}^{-1}$.

For the investigation of the role of solvent permittivity it is important to realize that, following eqn (9), the key control parameter is the dimensionless electrostatic coupling, $u=l_{\mathrm{B}} / b$. In our simulations $b$ is fixed based on an estimate from the geometry of poly(acrylic acid), while for other polyelectrolytes the value could be different depending on the chemical structure. For example for poly(styrene sulfonate) distance between the charged groups is about $1 \mathrm{~nm}$ because they are attached to a rather bulky side-group. ${ }^{48}$ Such details are below the resolution of the coarse-grained model, but should be considered when comparing simulation results to experiments. Therefore comparison of simulations with experiments should always be done for the given value of $u$, rather than $b$ or $\varepsilon_{\mathrm{r}}$ alone. For example the case of poly(styrene sulfonate) in water with $l_{\mathrm{B}} / b \approx 0.7$ could be mapped on our simulation results with $\varepsilon_{\mathrm{r}} \approx 140$ and $b=0.41 \mathrm{~nm}$. To allow for such mapping we vary the permittivity in the range $17<\varepsilon_{\mathrm{r}}<293$ which goes slightly beyond the highest available permittivities of real solvents, $\varepsilon_{\mathrm{r}} \approx 180 .^{49}$ It should be noted that such re-scaling of electrostatic interactions neglects the excluded volume effects due to finite size of ions and of the polymer segments. In most situations considered here, the excluded volume is negligible because typical distances between ions are much greater than their own size. Only beyond the Manning condensation threshold, when many counterions are condensed, the excluded volume contribution may become important and render the abovedescribed mapping inapplicable.

To rule out possible artifacts of periodic boundary conditions and finite size effects due to small number of chains in the box, for selected systems we compared simulations with $n=\{1,3,5,10\}$ chains per simulation box, adjusting the box volume such as to keep the polymer concentration constant. In most cases, the results were rather insensitive to the number of chains in the box, the differences being smaller than the estimated statistical error (see Fig. S1 of the ESI, $\dagger$ for details). In some cases, however, we observed significant differences. These are addressed in the respective parts of Results and discussion.

\section{Simulation methodology}

To account for the ionization reaction we employ the reaction ensemble, ${ }^{50}$ and to sample the configuration space we use a variant of the hybrid Monte Carlo (HMC) $\operatorname{method}^{51}$ particularly suitable to polymer systems. ${ }^{52}$ In each HMC step we perform either a conformation move, or a reaction move. In the conformational move the new state is obtained by a dynamical time evolution as in the usual molecular dynamics (except for using a modified evolution Hamiltonian ${ }^{52}$ ), and the trial configuration thus obtained is accepted or rejected using the Metropolis criterion (with the original Hamiltonian). The freely adjustable parameters (time step, trajectory length, etc.) are optimized to minimize the number of force evaluations per one independent sample of radius of gyration of the chain. The optimum is a compromise between faster evolution and better energy conservation entering the Metropolis criterion. It allows for much longer time-steps than in ordinary molecular dynamics while still exactly sampling the Boltzmann distribution. In the reaction move we select with equal probabilities the forward or reverse direction of the reaction in eqn (1). In the forward direction a randomly selected non-ionized monomer becomes ionized, and an $\mathrm{H}^{+}$ion is inserted at a random position in the box. In the reverse direction a randomly selected ionized monomer is de-ionized, and a randomly selected $\mathrm{H}^{+}$ion is removed. The acceptance probability $P^{\xi}$ for move from state o to $\mathrm{n}$ in the reaction ensemble is given by the criterion: ${ }^{50}$

$$
P^{\xi}=\min \left(1, V^{\bar{\nu} \xi} \Gamma^{\xi} \mathrm{e}^{-\beta \Delta U} \prod_{i=1} \frac{N_{i}^{0} !}{\left(N_{i}^{0}+\nu_{i} \xi\right) !}\right),
$$

where $\Delta U=U_{\mathrm{o}}-U_{\mathrm{n}}$ is the interaction energy change, $1 / \beta=k_{\mathrm{B}} T, V$ is the simulation box volume, $\bar{\nu}=\sum_{i} \nu_{i}$, and $\nu_{i}$ is the stoichiometric coefficient of species $i$ in the reaction eqn (1) (negative for the reactants and positive for the products). The instantaneous number of species $i$ in the simulation box, $N_{i}$ is linked to its the initial number, $N_{i}^{0}$ through the extent of reaction, $\xi$ :

$$
N_{i}=N_{i}^{0}+\nu_{i} \xi
$$

Finally, $\Gamma$ is related to $K_{\mathrm{A}}$ as

$$
\Gamma=K_{\mathrm{A}}\left(\frac{p^{\ominus}}{N_{\mathrm{A}} k_{\mathrm{B}} T}\right)^{\bar{\nu}},
$$

where $N_{\mathrm{A}}$ is the Avogadro number and $p^{\ominus}=1$ atm is the arbitrarily chosen standard pressure. At low densities considered here this simple method without any bias is sufficiently efficient. A detailed description can be found in our previous work. ${ }^{25}$

\subsection{Simulation protocol and data analysis}

Starting from an arbitrary polymer conformation, we first perform an equilibration run which is discarded from further analysis, followed by a longer production run which is used for sampling and collection of data. Duration of a typical simulation is $10^{5}$ configuration moves and about the same number of reaction moves. Per configuration move, we perform an MD simulation run of about 50 time steps. The HMC method yields correct ensemble averages irrespective of the choice of the above parameters, however, their values dramatically influence the efficiency of sampling. The optimum parameter range is relatively narrow and system-dependent, therefore it needs to be determined by tuning.

To characterize the quality of our data, we use the method of Wolff $^{53}$ to estimate the number of statistically independent samples. Our typical productive run generates about $10^{4}$ uncorrelated samples of $R_{\mathrm{g}}$, which turns out to be the slowest evolving studied observable. The evolution of some systems (low $\varepsilon_{\mathrm{r}}$, large $N$, low or high $\alpha$ ) was slower, so that it was difficult 
to obtain the desired number of uncorrelated samples. We consider $10^{3}$ uncorrelated samples as a threshold below which the simulations were not credible. In the reaction ensemble it is necessary to ensure that both the ionization and the polymer conformations are sampled properly by tuning the reaction step probability so that ideally the autocorrelation times of $\alpha$ and $R_{\mathrm{g}}$ are comparable. In practice, the reaction step is computationally much cheaper than the conformational move, therefore we prefer to oversample the reaction using a higher reaction probability. Then the overall quality of the simulation can be assessed on the basis of the slower conformational evolution alone at the cost of small computational overhead.

\section{Results and discussion}

\subsection{Local pH and bulk pH}

In Fig. 1 we show the density profiles of $\mathrm{H}^{+}$ions around the polymer chain with $\alpha \approx 1$, as a function of distance from the middle monomer for a series of polymer concentrations. The distance in Fig. 1 is scaled by the typical separation between the chains, $R_{\mathrm{cc}}$, which can be estimated as $R_{\mathrm{cc}}=\left(3 N / 4 \pi c_{\mathrm{pol}}\right)^{1 / 3}$. As a general trend, the concentration profiles initially decay with the power law close to the theoretical prediction $c(r) \sim r^{-2}$ for star-like polyelectrolytes. ${ }^{54}$ The same power law is even more closely followed by the segment density profile (see ESI, $\uparrow$ Fig. S2), indicating uniformly stretched chains. A qualitative difference shows up at higher separations, where the segment density profiles rapidly drop to unity while $\mathrm{H}^{+}$profiles level off in the intermediate region between the chains. Fig. 1 shows profiles obtained with 5 chains in the box in order to demonstrate that in this region the profiles pass through a minimum which corresponds to half of the maximum separation between two chains. Then they increase again as the distance approaches typical separation between two chains, with the highest probability of

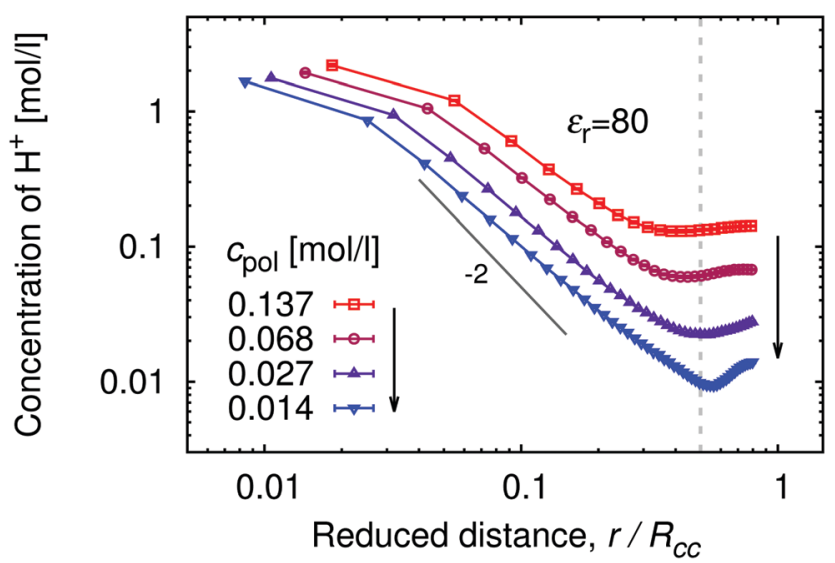

Fig. 1 Proton concentration as a function of distance from the central monomer $r$, scaled by the average separation between the chains $R_{\mathrm{cc}}$, obtained with 5 chains per simulation box for fully ionized chains with $K_{\mathrm{A}}=10,(\alpha=1)$, solvent permittivity $\varepsilon_{\mathrm{r}}=80$ and a series of polymer concentrations, $c_{\text {pol }}$, given in the legend. The vertical dashed line marks the distance where $r / R_{\mathrm{cc}}=1 / 2$. finding another polymer chain (for better illustration, see also simulation snapshot in the ESI, $\dagger$ Fig. S9). In principle, we should observe further oscillations of the concentration profile with decreasing amplitude, similar to the shape of radial distribution functions of simple liquids. However, this would require far more chains in the simulation box than we can conveniently handle. Notably, the difference between $c_{\mathrm{H}^{+}}$near the chain and in the bulk (minimum of $c_{\mathrm{H}^{+}}(r)$ ), makes a factor of $\approx 5$ for the high polymer concentrations $c_{\text {pol }} \approx 1.4 \times$ $10^{-1} \mathrm{~mol} \mathrm{l}^{-1}$, and a factor of $\approx 50$ for the low polymer concentrations $c_{\text {pol }} \approx 1 \times 10^{-2} \mathrm{~mol} \mathrm{l}^{-1}$.

The concentration profiles of $\mathrm{H}^{+}$ions are determined by the potential of mean force, $\tilde{U}(r)$, which is the effective interaction felt by an ion at a given position, averaged over positions of all other particles in the system. ${ }^{55} \tilde{U}(r)$ is defined as the negative logarithm of the pair correlation function, $g(r)$, and can be approximated by the mean electrostatic potential, $\langle\Psi(r)\rangle$. For the distribution of $\mathrm{H}^{+}$ions around the chain we have $g(r) \sim c_{\mathrm{H}^{+}}(r)$, hence (up to an arbitrary additive constant)

$$
\tilde{U}(r)=-k_{\mathrm{B}} T \ln \left(\left\langle c_{\mathrm{H}^{+}}(r)\right\rangle\right) \approx\langle\Psi(r)\rangle .
$$

The approximation $\tilde{U}(r) \approx\langle\Psi(r)\rangle$ has been used in recent experimental works to determine the local electrostatic potential near a polyelectrolyte chain from the measured local concentration of $\mathrm{H}^{+}$ions. ${ }^{16-18}$ In simulations we can explicitly calculate $\langle\Psi(r)\rangle$ by inserting a test charge and a homogeneously distributed background charge ${ }^{56,57}$ and averaging over different configurations. At the same time, we can calculate $\tilde{U}(r)$ from the concentration profiles shown in Fig. 1. The comparison of $\tilde{U}(r)$ and $\langle\Psi(r)\rangle$ in Fig. 2 shows very good agreement almost in the whole range. There is a slight disagreement at separations corresponding to the maximum probability of finding another chain, around $r / R_{\mathrm{cc}}=1 / 2$. Here $\tilde{U}(r)$ exhibits a weak maximum which is most visible at the lowest concentration, while $\langle\Psi(r)\rangle$ is

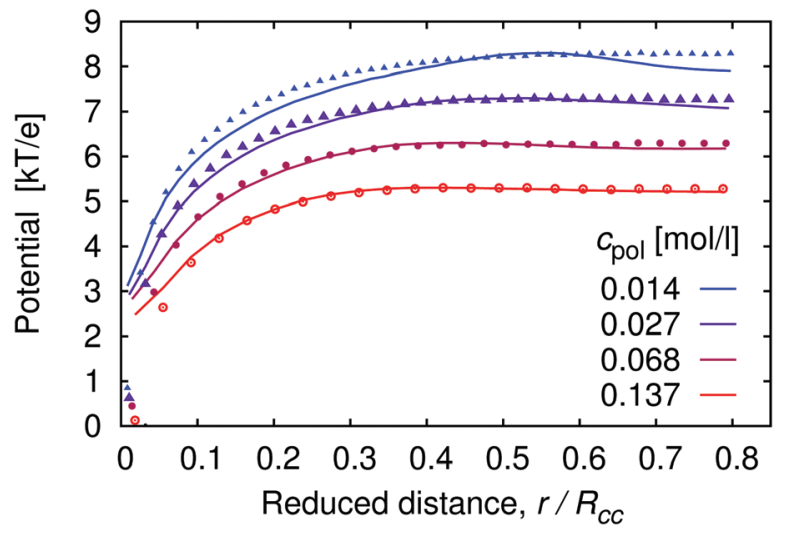

Fig. 2 Effective potential felt by $\mathrm{H}^{+}$ions as a function of distance from the central monomer $r$, scaled by the average separation between the chains $R_{\mathrm{cc}}$, for the same systems as in Fig. 1. Points represent the mean electrostatic potential $\langle\Psi(r)\rangle$, curves represent the potential of mean force $\tilde{U}(r)$ (see eqn (18)). We add an arbitrary constant to each potential such that the maxima of the corresponding potentials at the same $c_{\text {pol }}$ attain the same value. 
quite flat. Around $r=R_{\mathrm{cc}}$ the angular dependence of $\langle\Psi(r)\rangle$ is very strong: deep minima in very localized positions where the other chains are found, flat maxima elsewhere. With strong angular correlations, the mean-field averaging over angular coordinates is not well applicable. Another disagreement occurs at very small values of $r \leq \sigma$ where the short-ranged excluded volume interaction dominates over electrostatics. However, for the experimental estimation of $\langle\Psi(r)\rangle$ from the local proton concentration the relevant quantity is the difference $\langle\psi(\sigma)\rangle-\left\langle\psi\left(R_{\mathrm{cc}} / 2\right)\right\rangle$, in which case the above-discussed differences are quite inconsequential. Therefore we can conclude that the recent experiments ${ }^{16-18}$ provide a very good approximation the true value of $\langle\Psi(r)\rangle$.

As discussed in the section Theoretical background, the concentration of $\mathrm{H}^{+}$ions in the vicinity of the chain determines the local $\mathrm{pH}$, while $c_{\mathrm{H}^{+}}$far away from the chain (at the minimum of the $c_{\mathrm{H}^{+}}(r)$ dependence) determines the bulk $\mathrm{pH}$ or simply $\mathrm{pH}$ (i.e., the value that would be measured by a conventional $\mathrm{pH}$ meter). For the local pH near the chain we use the maximum of the $c_{\mathrm{H}^{+}}(r)$ dependence, which typically occurs within $r \lesssim 2 b$. Since our definition of local $\mathrm{pH}$ is arbitrary, we performed a sensitivity analysis to demonstrate that various plausible definitions yield very similar results (see ESI, $\dagger$ Fig. S3). Fig. 3 shows that local $\mathrm{pH}$ is always lower than the bulk $\mathrm{pH}$. At this point it is important to recall that in our simulations all the $\mathrm{H}^{+}$ions come from the polyelectrolyte ionization and the different $\mathrm{pH}$ values are attained by means of varying the $\mathrm{p} K_{\mathrm{A}}$. Therefore low $\mathrm{pH}$ corresponds to high ionization and high $\mathrm{pH}$ corresponds to low ionization. In an experiment where one would use a polymer with a fixed $\mathrm{p} K_{\mathrm{A}}$ and control $\mathrm{pH}$ by a buffer, the situation would be reversed.

The biggest differences between the bulk and local $\mathrm{pH}$ occur when the polymer is fully ionized, while with decreasing ionization (increasing $\mathrm{pH}$ ) the local and bulk $\mathrm{pH}$ approach each other. In terms of proton concentration, this means that $c_{\mathrm{H}^{+}}$in the vicinity of the fully ionized chain is much higher than in the bulk. As the ionization of the chain decreases (increasing $\mathrm{pH}$ ), influence of the chain on the concentration profile becomes weaker, and local $\mathrm{pH}$ approaches the bulk value. This observation is consistent with existing experimental observations for the cationic poly(2-vinyl pyridine). ${ }^{18,46}$ In addition, our simulations show that the difference between the bulk and local $\mathrm{pH}$ significantly diminishes with increasing polymer concentration (Fig. 3) and increases with chain length (ESI, $\dagger$ Fig. S3). The concentration dependence of ionization can be understood by comparison with the concentration-dependence of profiles of $\mathrm{H}^{+}$ions in Fig. 1. With increasing dilution, the volume per chain increases, and counterions are distributed over the whole volume, while the chain connectivity restricts the volume in which ionized segments are confined.

Fig. 3 also reveals a small but still measurable increase in local $\mathrm{pH}$ when going from the middle of the chain (i.e., central monomer) toward the end (terminal monomers). This decrease in the concentration of counterions around the ends of polyelectrolyte chain has been termed end-effect. ${ }^{58}$ Similar trend was observed in experiments on star-like polyelectrolytes. ${ }^{18}$ Thanks to the higher molecular weight and star-like nature of

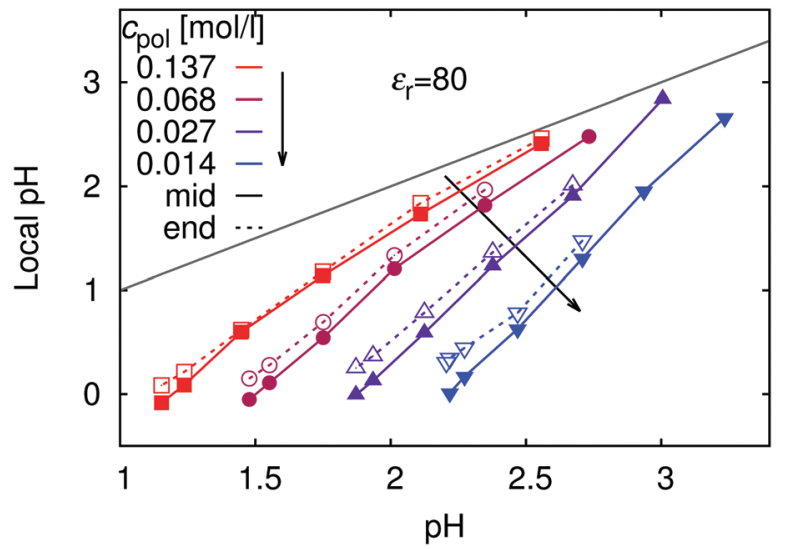

Fig. 3 Local pH (see eqn (8) for definition) in the vicinity of the chain: in the middle of the chain (full symbols) and at the end (empty symbols), as a function of $\mathrm{pH}$ in the bulk for various polymer concentrations, $c_{\text {pol- }}$. The grey line marks the values where both quantities are equal. Estimated errors are smaller than the symbol size.

the polymer the experimentally observed difference between the middle and the end was more pronounced (about 0.3 units of $\mathrm{pH}$ ) than in our simulations. However, our present results cannot explain the relation between the local and bulk $\mathrm{pH}$ in the strong poly(styrene sulfonate), ${ }^{17,45}$ where the ionization is independent of $\mathrm{pH}$ and the mechanism behind the local $\mathrm{pH}$ variation is presumably different.

\subsection{Influence of chain length}

To investigate the role of further parameters influencing the ionization behaviour of weak PEs, we performed series of simulations with various chain lengths and polymer concentrations. We observed significant artifacts of periodic boundary conditions for $N<10$ with single chain per simulation box. Therefore we fixed total number of monomer units per simulation box, such that $n N=100$. For $N \geq 10$ the difference between $n=10$ and $n=1$ was negligible at the given polymer concentration $c_{\text {pol }}=0.014 \mathrm{M}$.

In Fig. 4 we show the titration curves for chain lengths $1<N<200$. With $N=1$ (single monomer) we match the ideal titration curve (eqn (6)). As we increase the chain length, the titration curves start to deviate from the ideal one, gradually moving to higher $\mathrm{pH}$ with respect to the ideal titration curve. Because the titration curves are not only shifted but also deformed with respect to the ideal one, it is clear that it is impossible to characterize the non-ideality of a polyelectrolyte chain by a single value of $\mathrm{p} K_{\text {eff }}$. Instead, $\mathrm{p} K_{\text {eff }}$ becomes a parameter which depends on the degree of ionization of the chain, its length, concentration, etc. However, from $N \approx 50$ the deviation from the ideal behaviour stays constant, nearly independent of the chain length. Therefore we claim that the shift and deformation of the titration curve is mostly a local effect of the nearest few monomer units along the chain contour. This observation is consistent with the earlier simulations of Ullner et al., who observed a very weak dependence $\left(\mathrm{p} K_{\text {eff }}-\mathrm{p} K_{\mathrm{A}}\right) \sim \alpha^{1 / 3}(\ln N)^{2 / 3}$ in the long chain limit. ${ }^{29}$ Fitting our data by eqn (11) with $m$ as 


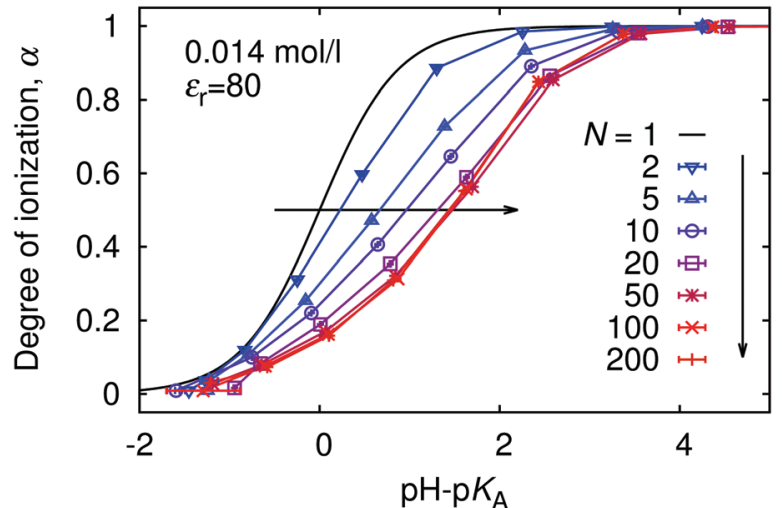

Fig. 4 The titration curves for various lengths of polymer chains, $N$.

adjustable fit parameter we observe that this equation fairly well captures the shape of the deformed titration curves for various chain lengths (see ESI $\dagger$ Fig. S4). Interestingly, when we used eqn (9) with all its numerical pre-factors, the obtained value of $m$ was very different from that from the fit, and the predicted curve was shifted to much higher values of $\mathrm{pH}$, entirely outside the range of Fig. 4.

\subsection{Influence of polymer concentration}

The relation between the polymer concentration and the local concentration of $\mathrm{H}^{+}$is further manifested in the concentrationdependence of the titration curves, shown in Fig. 5. We again observe the general trend that the titration curves are shifted to higher values of $\mathrm{pH}$ with respect to the ideal titration curve. The striking feature of this figure is that deviations from the ideal titration curve increase with dilution, quite opposite to the behaviour of simple acids, which become ideal when diluted. This observation is qualitatively consistent with increasing $\mathrm{p} K_{\text {eff }}$ observed in the titration of both poly(acrylic acid) and poly(methacrylic acid). ${ }^{12,13}$ To understand this phenomenon we recall that the leading contribution to the deviation originates from electrostatic repulsion of nearby ionized groups. The counterions surrounding the chain screen this repulsion.

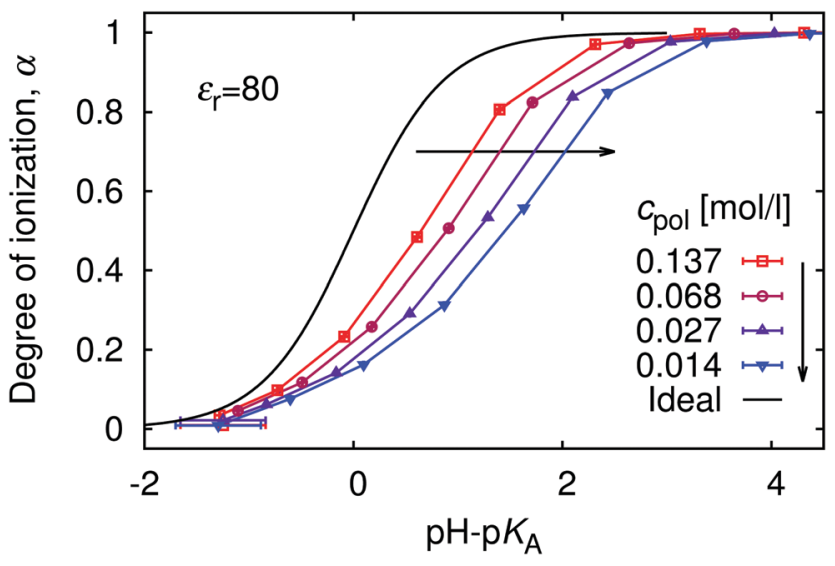

Fig. 5 The titration curves at various polymer concentrations, $c_{\text {pol. }}$ The black solid line is the ideal titration curve.
However, with increasing polymer dilution the counterions are distributed over bigger volume, and their concentration near the chain drops ( $c f$. Fig. 1), while the separation of ionized polymer segments remains practically the same. The lower concentration of counterions leads to weaker screening, and hence stronger repulsion of the bare charges on the chain. Ultimately, the stronger repulsion leads to suppressed ionization.

Since the deviation from ideal titration increases with dilution, it might provide an explanation why eqn (9) largely overestimates the shift: it has been derived neglecting the role of counterions, effectively assuming infinite dilution. To investigate this further, in Fig. 6 we re-plot the data from Fig. 5 in the form of the universal titration curve, eqn (11). In this representation we observe that only at low $\alpha$ the dependence is linear, in accordance with eqn (9) and (11), while above $\alpha \approx 0.5$ the simulation results deviate from the predicted linear dependence. In addition, the slope of the plots, which determines the parameter $m$, increases with dilution, so that at extreme dilution it might indeed reach the value predicted by eqn (9). The deviation from linearity beyond $\alpha=0.5$ could be attributed to the Manning condensation and strong correlations between the chain and its counterions. The Manning condensation is not captured by the theory of Katchalsky and Gillis and we will further address this point in the Section 5.4. We remark that for our system with flexible macromolecule of finite length and with inhomogeneous distribution of point charges the onset of Manning condensation is a gradual transition, as discussed by Mann et al. ${ }^{59}$ It can be identified from the plot of electrostatic energy per particle as a function of the Manning parameter (see ESI, $\dagger$ Fig. S5). In our case the plot features a broad flat maximum. For an infinitely long homogeneously charged rod the Manning condensation features a second order transition which can be identified by a cusp in the dependence of the electrostatic energy per particle on the Manning parameter. ${ }^{60,61}$

The complex shape of titration curves in Fig. 5 is a consequence of the interplay between the ionization of the polymer, and the accompanying conformational changes, illustrated by simulation snapshots in Fig. 7, and further by the dependence

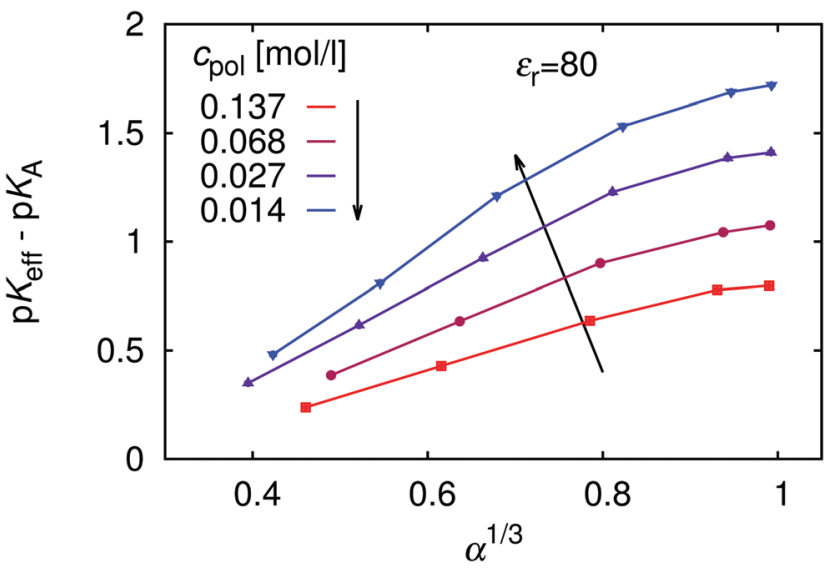

Fig. 6 The universal titration curve compared with our simulation data for various polymer concentrations, $c_{\text {pol }}$. 

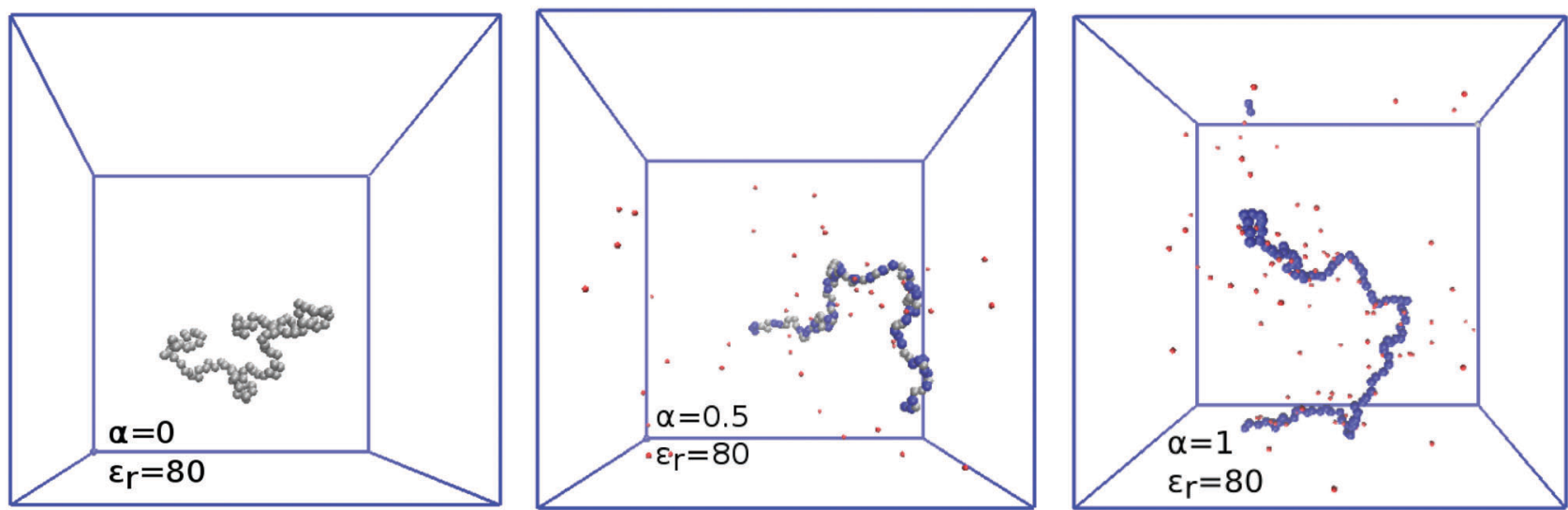

Fig. 7 Simulation snapshots showing polymer conformations at various degrees of ionization $\alpha$. From left to right: $\alpha=0,0.5$, and 1 . Colour code: silver uncharged monomer, blue - charged monomer, red $-\mathrm{H}^{+}$ion.

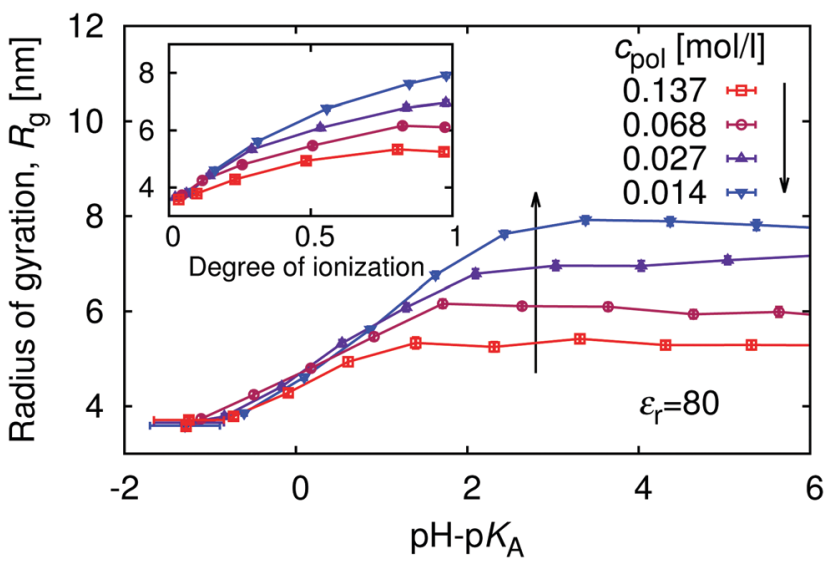

Fig. 8 Variation of the radius of gyration, $R_{\mathrm{g}}$, with $\mathrm{pH}$.

of the radius of gyration, $R_{\mathrm{g}}$, on the degree of ionization in the insert of Fig. 8. Here we observe that the chain conformation changes from the swollen coil at $\alpha \approx 0$ to the nearly rod-like conformation at $\alpha \approx 1$. At the expense of entropy loss, the chain compensates in this way for the increased electrostatic repulsion at higher ionization. Furthermore, we observe in Fig. 8 that the maximum expansion of the chain increases with decreasing polymer concentration. Again, this can be understood when we recall from Fig. 1 that with decreasing $c_{\mathrm{pol}}$ the concentration of $\mathrm{H}^{+}$decreases in the whole range, so that the screening of charges on the chain vanishes. With weaker screening, the expansion due to electrostatic repulsion of the ionized groups is stronger. Fig. 8 also demonstrates that polymer segments are confined to a narrow region of the chain, while counterions are diluted: upon ten-fold decrease of concentration, the change of $R_{\mathrm{g}}$ at full ionization amounts to less than $50 \%$. When $R_{\mathrm{g}}$ is plotted as a function of $\mathrm{pH}-\mathrm{p} K_{\mathrm{A}}$ in Fig. 8, it appears to increase almost independently of $c_{\mathrm{pol}}$ up to a certain value of $\mathrm{pH}$ where the expansion stops. This value depends on the polymer concentration, and from Fig. 5 we can see that it corresponds to the point where $\alpha$ approaches unity. Interestingly, comparison of Fig. 5 and 8 reveals that the nearly-matching values of $R_{g}$ at the same $\mathrm{pH}$ but different polymer concentrations actually correspond to different degrees of ionization, $\alpha$. The near coincidence of the $R_{\mathrm{g}}\left(\mathrm{pH}-\mathrm{p} K_{\mathrm{A}}\right)$ curves thus demonstrates an interesting cancellation of two non-ideal effects.

Last but not least, it is interesting to realize that as the polymer expands with increasing $\alpha$, its overlap concentration, $c^{*} \sim N / R_{\mathrm{g}}{ }^{3}$, decreases significantly (see ESI, $\dagger$ Fig. S6). Even if solution of the neutral polymer is prepared well below the overlap concentration, such that individual chains do not affect each other, as its conformation changes in the course of a titration experiment, the overlap concentration decreases and the fully ionized chains may significantly overlap and affect each other.

\subsection{Influence of solvent permittivity}

Replacing the solvent by another one with a different permittivity, or by continuous variation of composition of mixed solvents, it is possible to change the relative permittivity of the medium, and in this way to tune the electrostatic coupling strength $u=l_{\mathrm{B}} / b$. Within a narrower range, electrostatic coupling can also be varied by using a polymer with different separation of the charged groups.

An increase in electrostatic coupling increases the enthalpy gain as the ions condense on the chain, while the corresponding entropy loss remains the same. This interplay of enthalpy and entropy has a non-trivial effect on the conformation, manifested in the non-monotonic dependence of $R_{\mathrm{g}}$ on $u$ at fixed $\alpha$ in Fig. 9 . At $u \lesssim 1$ (high $\varepsilon_{\mathrm{r}}$ ), typical for polar solvents, entropy dominates the behaviour of counterions. They are only weakly coupled to the chain, and contribute to the overall picture only through screening of the interactions between the charged monomers. At the same time, mutual repulsion between charges on the chain increases with $u$, therefore the chain expands and $R_{\mathrm{g}}(u)$ increases with $u$. Around the Manning condensation threshold, $u \approx 1$, the ions start to condense, $R_{\mathrm{g}}(u)$ passes trough a maximum and starts to decrease again at higher $u$. At high coupling, the counterions become highly correlated with the chain. The alternating pattern of positive and negative charges along the chain brings about a net attractive force, termed the counterion-induced correlation attraction, ${ }^{62,63}$ which makes the polyelectrolyte shrink if $u$ is further increased. This is well illustrated in Fig. 10 by simulation snapshots of the polymer with $\alpha=1.0$ at different electrostatic 
Relative permittivity, $\varepsilon_{\mathrm{r}}$

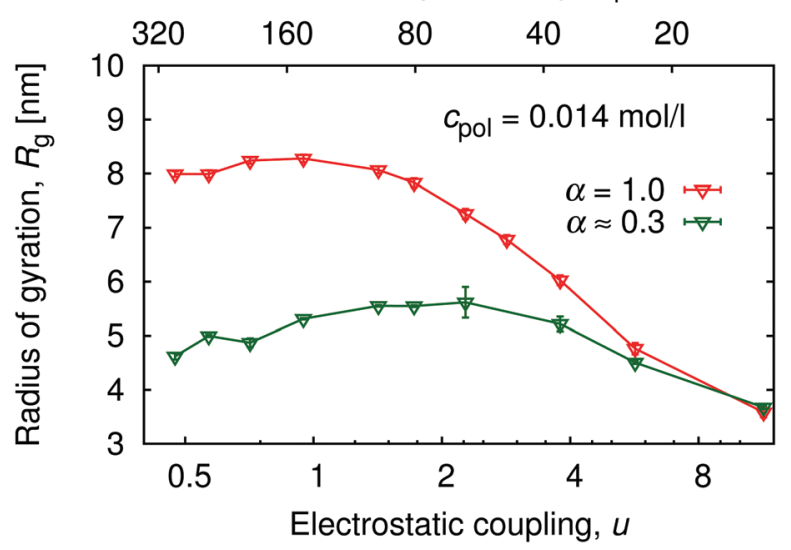

Fig. 9 Variation of the radius of gyration, $R_{\mathrm{g}}$, with electrostatic coupling strength (bottom axis), and solvent permittivity, $\varepsilon_{\mathrm{r}}$ (top axis) at two degrees of ionization: $\alpha=1.0$ and $\alpha=0.3 \pm 0.08$. See also ESI, $\dagger$ Fig. S 8 for the complete data set of $R_{\mathrm{g}}\left(\alpha, \varepsilon_{\mathrm{r}}\right)$.

couplings: with increasing $u$ an increasing portion of counterions is located in the immediate vicinity of the chain, and the chain extension decreases. The correlation effects require the discrete character of individual charged monomers and counterions to be explicitly considered, beyond the mean-field picture of the polyelectrolyte as a homogeneously charged worm-like chain in a cloud of counterions. For the same reason, if the electrostatic coupling is re-scaled by the degree of ionization as $\alpha u$, the two curves of $R_{\mathrm{g}}(\alpha u)$ in Fig. 9 for $\alpha=0.3$ and 1.0 do not collapse on a single universal master curve.

The interplay between the chains and counterions at various permittivities can be also illustrated by distribution of counterions along the chain. Here we use the approach of Limbach et al. ${ }^{58,64}$ who defined the distance of a given counterion from the chain as the smallest distance between that ion and any monomer unit. At long distances this function behaves indistinguishably from the conventional pair correlation function, $g(r)$, up to $r / R_{\mathrm{cc}} \approx 1 / 2$, where it sharply drops to zero. At short distances, however, it respects the local symmetry of the

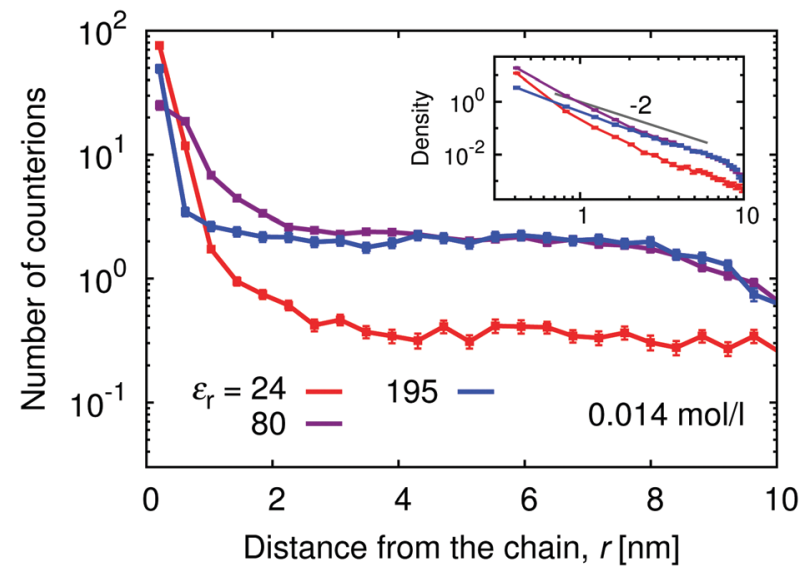

Fig. 11 Distribution of counterions along the polyelectrolyte chain for different relative permittivities, $\varepsilon_{r}$, at $\alpha=1$.

problem: near the chain the distribution has local cylindrical symmetry while on long distances it is approximately spherical.

Above the counterion condensation threshold the condensed counterions compensate for the excess charge on the chain and effectively renormalize the coupling parameter to $u \approx 1$. Therefore at $\alpha \approx 1$ the counterion condensation should not occur at $\varepsilon_{\mathrm{r}} \gtrsim 160$, while at lower permittivities the number of condensed counterions should increase. We could estimate the number condensed counterions from the deviation of the plot in Fig. 11 in the vicinity of the chain from the plateau value at intermediate $r$. Note that this plateau only shows up at sufficiently low polymer concentrations (see ESI, $\dagger$ Fig. S7). At $\varepsilon_{\mathrm{r}}=195$ we observe that the curve is flat and only a few extra counterions are found in the immediate vicinity. With increasing coupling (decreasing $\varepsilon_{\mathrm{r}}$ ) the positive deviation at low separations becomes more significant, as the counterions accumulate around the chain and fewer of them are found in the plateau region at higher separations.

The counterion condensation has a profound influence on the local pH near the chain, as follows from Fig. 12. The slopes of the dependencies of local $\mathrm{pH}$ on $\mathrm{pH}$ in the bulk are much
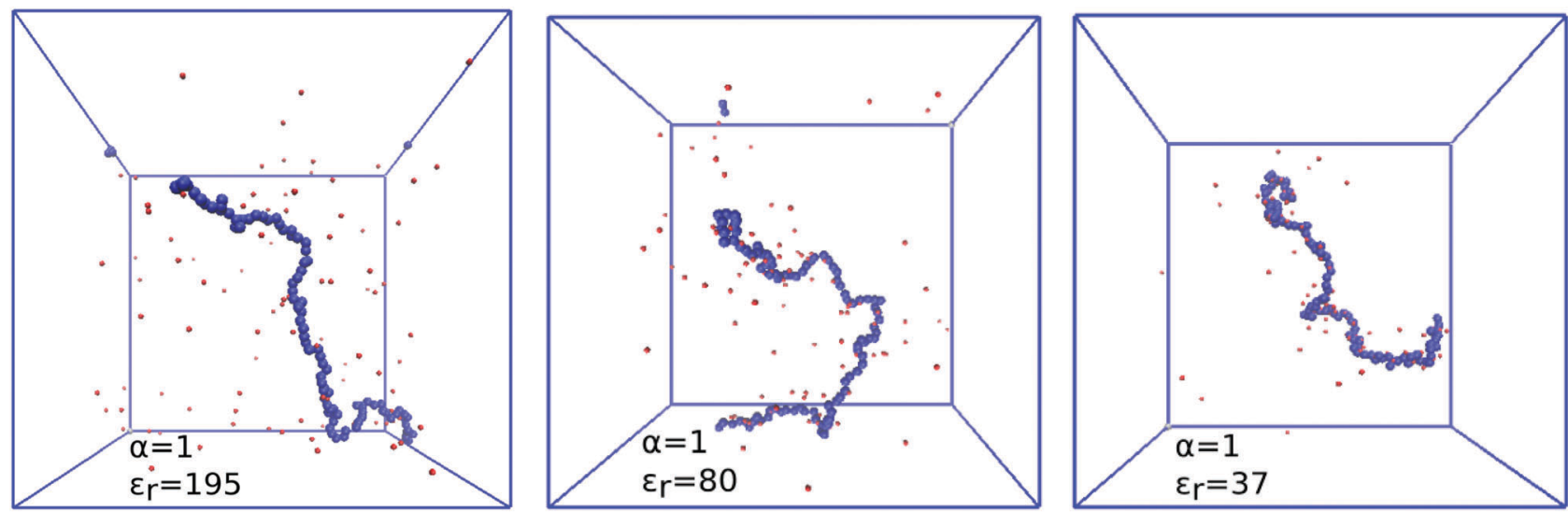

Fig. 10 Simulation snapshots showing counterion distribution and counterion condensation, from left to right: $\varepsilon_{\mathrm{r}}=195,80$, 37, which correspond to $u=0.7,1.7$ and 3.8. Colour code: silver - uncharged monomer, blue - charged monomer, red $-\mathrm{H}^{+}$ion. 


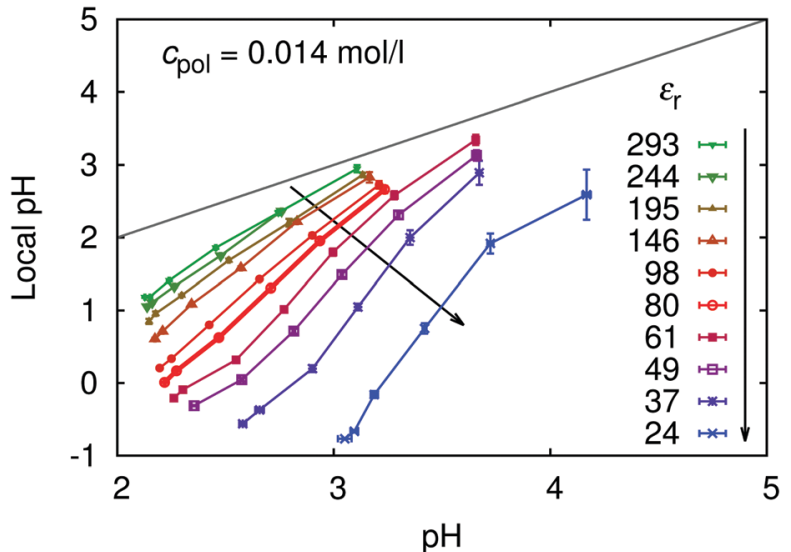

Fig. 12 Local $\mathrm{pH}$ in the vicinity of the chain, as a function of $\mathrm{pH}$ in the bulk for various solvent permittivities, $\varepsilon_{\text {r }}$.

steeper at lower permittivities (stronger coupling), while at higher permittivities (weaker coupling) the local and bulk $\mathrm{pH}$ do not differ too much.

Since ionization behaviour of a weak polyelectrolyte is intimately coupled to its conformation, the profound differences in conformations and distribution of small ions which occur with varying permittivity, significantly affect the titration curves, as is shown in Fig. 13. As the electrostatic coupling increases
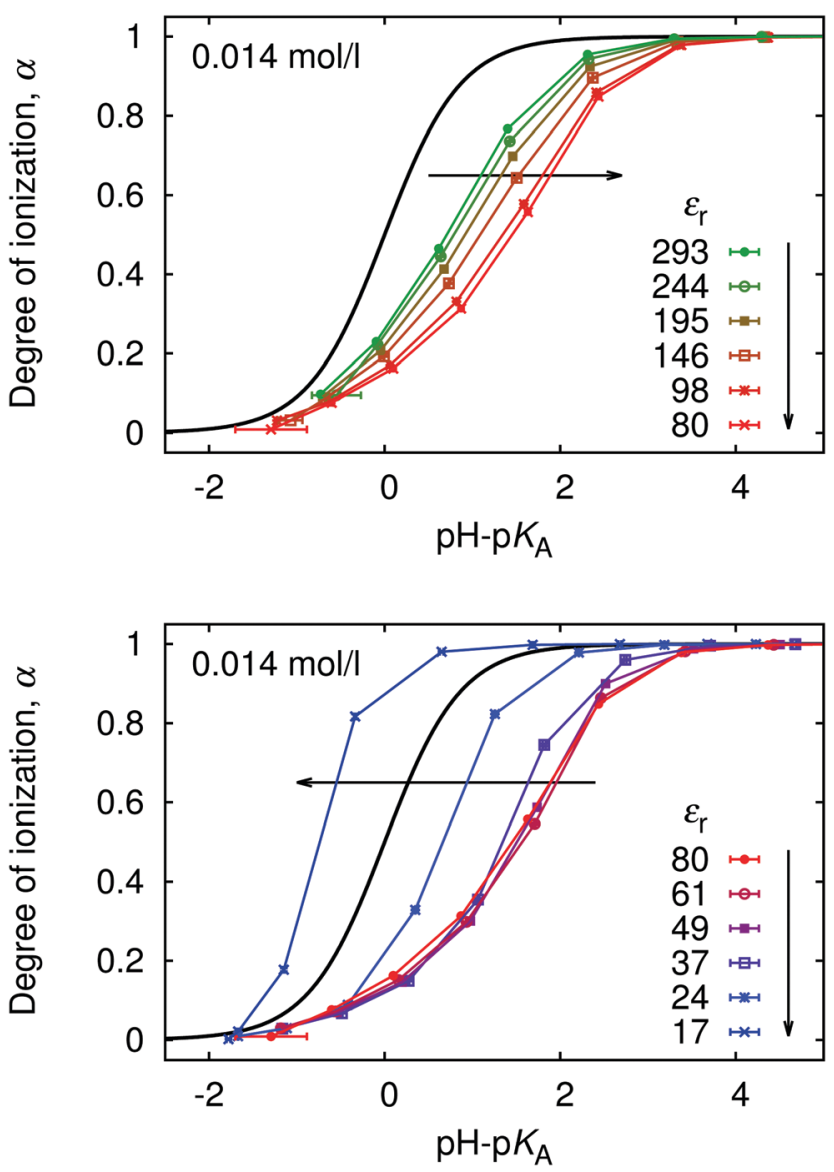

Fig. 13 The titration curves for various permittivities, $\varepsilon_{r}$.

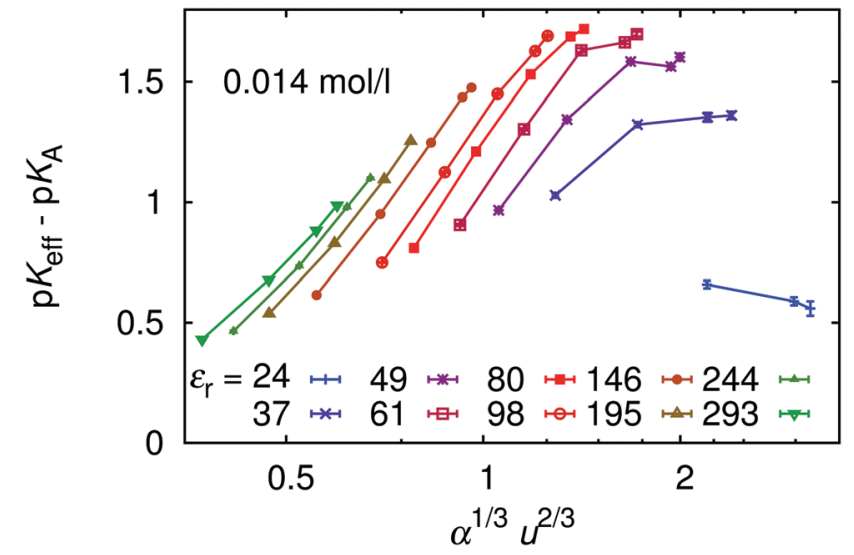

Fig. 14 The universal titration curve compared with our simulation data for different permittivities, $\varepsilon_{r}$.

up to $u \approx 2\left(\varepsilon_{\mathrm{r}} \approx 80\right)$, the curves move towards higher values of $\mathrm{pH}-\mathrm{p} K_{\mathrm{A}}$ and are increasingly deformed with respect to the ideal titration curve. At higher $u$ the trend reverses, and the curves approach the ideal titration curve and even move to the opposite side of it at very low $\varepsilon_{\mathrm{r}}$. This counter-intuitive phenomenon is not surprising when we consider the counterion-induced attraction at high $u$, which reduces the free energy cost of additional ionization. This observation is consistent with earlier work of Panagiotopoulos, ${ }^{32}$ who also observed that at very high $u$ the polymer titration curve can be even shifted to the other side of the ideal one. It remains an open question, however, whether such total reversal of the behaviour could be observed in experiments. Real polymers exhibit specific polymer-counterion interactions, which are very difficult to properly include in the coarse-grained modeling. These specific interactions would presumably favor precipitation of he polymer from solution under such conditions.

Finally, in Fig. 14 we plot our simulated titration curves at different $\varepsilon_{\mathrm{r}}$ as a function of $\alpha^{1 / 3} u^{2 / 3}$. Following eqn (11) these data should collapse on a single straight line. At low $u$ our data yield nearly straight lines but systematically shift to the right with increasing $u$ (decreasing $\varepsilon_{\mathrm{r}}$ ). At higher $u$ our curves are increasingly deformed, which is presumably the consequence of counterion condensation. Thus we conclude that the universal master curve from eqn (9) and (11) does not provide an appropriate prediction of the dependence of $\alpha$ on $\varepsilon_{\mathrm{r}}$ even at low electrostatic coupling, while it completely fails at high coupling. However, if counterion condensation is not too strong, eqn (11) can still be used to fit experimental data and the above failure translates to the dependence of fit parameters $\mathrm{p} K_{\mathrm{A}}$ and $m$ on $\varepsilon_{\mathrm{r}}$.

\section{Conclusion}

In this work we have performed coarse-grained simulations of weak polyelectrolytes, explicitly accounting for the ionization equilibrium by means of the reaction ensemble method. ${ }^{50}$ This approach allowed us to explicitly consider the coupling between the ionization and conformation of a model polyelectrolyte chain. Using the simulation data, we have discussed the 
differences between the concentration of $\mathrm{H}^{+}$ions in the bulk and in the immediate vicinity of the chain, commonly termed the "bulk pH" and the "local pH". We have shown that the bulk and local $\mathrm{pH}$ can easily differ by two or more units, consistent with recent experimental observations. ${ }^{18,46}$ Next, we have investigated how the polymer chain length and concentration affect the titration curves. We observed that beyond $N \approx 20$ the chain length effect is very weak which supports our claim that the non-ideal titration behaviour is mostly a local effect of the nearest groups on the chain. The decreasing polymer concentration results in an increasing non-ideality, opposite to the behaviour of simple acids which become ideal at infinite dilution. This counter-intuitive behaviour is consistent with experiments, ${ }^{12,13}$ and could be explained in terms of distribution of counterions and screening effects. Our investigation of the influence of electrostatic coupling strength (solvent permittivity) has revealed two regimes: at low coupling the non-ideality initially increases with decreasing $\varepsilon_{\mathrm{r}}$ as a consequence of increasing electrostatic repulsion. At high coupling the non-ideality decreases again and eventually changes the sign. At low coupling, the effect is dominated by increasing repulsion among the charged polymer segments, which is compensated at higher coupling by counterion condensation and effective attraction due to strong correlations between the chain and its ions. Finally, we have shown that simulation results at different permittivities exhibit systematic deviations from the expected universal master curve predicted by Katchalsky and Gillis, which is commonly used to fit experimental data.

\section{Acknowledgements}

We gratefully acknowledge Dr Oleg Rud for his discussions and comments to the manuscript. This research was supported by the Czech Science Foundation (grant P208/14-23288J) and the Ministry of Education, Youth and Sports (CUCAM CZ.02.1.01/0.0/0.0/ 15_003/0000417). Access to computing facilities of CERIT-SC (CZ.1.05/3.2.00/08.0144) and National Grid Infrastructure MetaCentrum (LM2010005) is acknowledged too.

\section{References}

1 M. A. Dyakonova, N. Stavrouli, M. T. Popescu, K. Kyriakos, I. Grillo, M. Philipp, S. Jaksch, C. Tsitsilianis and C. M. Papadakis, Macromolecules, 2014, 47, 7561-7572.

2 M. A. Dyakonova, A. V. Berezkin, K. Kyriakos, S. Gkermpoura, M. T. Popescu, S. K. Filippov, P. Štépánek, Z. Di, C. Tsitsilianis and C. M. Papadakis, Macromolecules, 2015, 48, 8177-8189.

3 O. Colombani, E. Lejeune, C. Charbonneau, C. Chassenieux and T. Nicolai, J. Phys. Chem. B, 2012, 7560-7565.

4 O. Borisova, L. Billon, M. Zaremski, B. Grassl, Z. Bakaeva, A. Lapp, P. Stepanek and O. Borisov, Soft Matter, 2012, 8, 7649.

5 O. V. Borisova, L. Billon, R. P. Richter, E. Reimhult and O. V. Borisov, Langmuir, 2015, 31, 7684-7694.

6 J. Xie, K. Nakai, S. Ohno, H.-J. Butt, K. Koynov and S. Yusa, Macromolecules, 2015, 48, 7237-7244.
7 K. Geisel, L. Isa and W. Richtering, Angew. Chem., Int. Ed., 2014, 53, 4905-4909.

8 A. J. Schmid, R. Schroeder, T. Eckert, A. Radulescu, A. Pich and W. Richtering, Colloid Polym. Sci., 2015, 293, 3305-3318.

9 C. S. Patrickios and T. K. Georgiou, Curr. Opin. Colloid Interface Sci., 2003, 8, 76-85.

10 E. J. Kepola, E. Loizou, C. S. Patrickios, E. Leontidis, C. Voutouri, T. Stylianopoulos, R. Schweins, M. Gradzielski, C. Krumm, J. C. Tiller, M. Kushnir and C. Wesdemiotis, ACS Macro Lett., 2015, 4, 1163-1168.

11 T. Swift, L. Swanson, M. Geoghegan and S. Rimmer, Soft Matter, 2016, 12, 2542-2549.

12 R. Arnold, J. Colloid Sci., 1957, 12, 549-556.

13 C. Heitz, M. Rawiso and J. François, Polymer, 1999, 40, 1637-1650.

14 A. Katchalsky and J. Gillis, Recl. Trav. Chim. Pays-Bas, 1949, 68, 879.

15 F. Plamper, H. Becker, M. Lanzendorfer, M. Patel, A. Wittemann, M. Ballauff and A. Müller, Macromol. Chem. Phys., 2005, 206, 1813-1825.

16 G. Xu, S. Luo, Q. Yang, J. Yang and J. Zhao, J. Chem. Phys., 2016, 145, 144903.

17 S. Wang, S. Granick and J. Zhao, J. Chem. Phys., 2008, 129, 241102.

18 C. Qu, Y. Shi, B. Jing, H. Gao and Y. Zhu, ACS Macro Lett., 2016, 402-406.

19 S. P. L. Sörensen, Carlsberg Laboratorien, Kopenhagen, 1909, vol. II, pp. 131-200.

20 I. N. Levine, Physical Chemistry, Mc Graw Hill, VI edn, 2009.

21 R. P. Buck, S. Rondinini, A. K. Covington, F. G. K. Baucke, C. M. A. Brett, M. F. Camoes, M. J. T. Milton, R. Mussini, R. Naumann, K. W. Pratt, P. Spitzer and G. S. Wilson, Pure Appl. Chem., 2002, 74, 2169.

22 H. J. Limbach, C. Holm and K. Kremer, Europhys. Lett., 2002, 60, 566-572.

23 P. Košovan, Z. Limpouchová and K. Procházka, Macromolecules, 2006, 39, 3458-3465.

24 E. Raphael and J. F. Joanny, Europhys. Lett., 1990, 13, 623.

25 F. Uhlík, P. Košovan, Z. Limpouchová, K. Procházka, O. V. Borisov and F. A. M. Leermakers, Macromolecules, 2014, 47, 4004-4016.

26 J. L. Lützenkirchen, J. V. Male, F. Leermakers and S. Sjöberg, J. Chem. Eng. Data, 2011, 1602-1612.

27 M. Borkovec, G. J. Koper and C. Piguet, Curr. Opin. Colloid Interface Sci., 2006, 11, 280-289.

28 M. Lund and B. Jönsson, Q. Rev. Biophys., 2013, 46, 265-281.

29 M. Ullner, B. Jönsson and P. Widmark, J. Chem. Phys., 1994, 100, 3365.

30 M. Ullner and C. E. Woodward, Macromolecules, 2000, 33, 7144-7156.

31 B. Jönsson, M. Ullner, C. Peterson, O. Sommelius and B. Södeberg, J. Phys. Chem., 1996, 100, 409-417.

32 A. Panagiotopoulos, J. Phys.: Condens. Matter, 2009, 21, 424113.

33 S. Uyaver and C. Seidel, Europhys. Lett., 2003, 64, 536-542. 34 S. Uyaver and C. Seidel, Macromolecules, 2009, 42, 1352-1361. 
35 T. Zito and C. Seidel, Eur. Phys. J. E: Soft Matter Biol. Phys., 2002, 8, 339-346.

36 M. Ullner, B. Jönsson, B. Söderberg and C. Peterson, J. Chem. Phys., 1996, 104, 3048-3057.

37 M. Ullner and B. Jönsson, Macromolecules, 1996, 29, 6645-6655.

38 S. Ulrich, A. Laguecir and S. Stoll, J. Chem. Phys., 2005, 122, 094911.

39 F. Carnal, S. Ulrich and S. Stoll, Macromolecules, 2010, 43, 2544-2553.

40 S. Edgecombe, S. Schneider and P. Linse, Macromolecules, 2004, 37, 10089-10100.

41 G. S. Longo, M. O. de la Cruz and I. Szleifer, Macromolecules, 2011, 44, 147-158.

42 G. S. Longo, M. O. de la Cruz and I. Szleifer, ACS Nano, 2013, 7, 2693-2704.

43 J. Ziebarth and Y. Wang, J. Phys. Chem. B, 2010, 114, 6225-6232.

44 J. D. Ziebarth and Y. Wang, Biomacromolecules, 2010, 11, 29-38.

45 S. Luo, X. Jiang, L. Zou, F. Wang, J. Yang, Y. Chen and J. Zhao, Macromolecules, 2013, 46, 3132-3136.

46 S. Wang and Y. Zhu, Soft Matter, 2011, 7, 7410-7415.

47 F. Uhlík, P. Košovan, E. B. Zhulina and O. V. Borisov, Soft Matter, 2016, 12, 4846-4852.

48 M. Vögele, C. Holm and J. Smiatek, J. Chem. Phys., 2015, 143, 243151.

49 R. A. Butler, PhD thesis, University of Florida, 1978.

50 W. R. Smith and B. Tříska, J. Chem. Phys., 1994, 100, 3019-3027.
51 S. Duane, A. Kennedy, B. J. Pendleton and D. Roweth, Phys. Lett. B, 1987, 195, 216-222.

52 A. Irbäck, J. Chem. Phys., 1994, 101, 1661-1667.

53 U. Wolff, Comput. Phys. Commun., 2004, 156, 143-153.

54 O. V. Borisov, E. B. Zhulina, F. A. Leermakers, M. Ballauff and A. H. E. Müller, in Self Organized Nanostructures of Amphiphilic Block Copolymers I, ed. A. H. E. Müller and O. Borisov, Springer Berlin Heidelberg, 2011, vol. 241, pp. 1-55.

55 D. A. McQuarrie, Statistical Mechanics, Harper Collins, New York, 1976.

56 V. Ballenegger, A. Arnold and J. J. Cerdà, J. Chem. Phys., 2009, 131, 094107.

57 J. S. Hub, B. L. de Groot, H. Grubmüller and G. Groenhof, J. Chem. Theory Comput., 2014, 10, 381-390.

58 H. J. Limbach and C. Holm, J. Chem. Phys., 2001, 114, 9674-9682.

59 B. A. Mann, C. Holm and K. Kremer, Macromol. Symp., 2006, 237, 90-107.

60 A. Naji and R. R. Netz, Phys. Rev. Lett., 2005, 95, 185703.

61 A. Naji and R. R. Netz, Phys. Rev. E: Stat., Nonlinear, Soft Matter Phys., 2006, 73, 056105.

62 Q. Liao, A. V. Dobrynin and M. Rubinstein, Macromolecules, 2006, 39, 1920-1938.

63 A. Naji, S. Jungblut, A. G. Moreira and R. R. Netz, Phys. A, 2005, 352, 131-170.

64 H. J. Limbach and C. Holm, J. Phys. Chem. B, 2003, 107, 8041-8055. 